Ditieri, M.; Cortese, G.B. y González Demaría, Y. G. Cuando la realidad supera la norma: gestación por sustitución y filiación post mortem. Los efectos de su omisión. Derecho y Ciencias Sociales. Abril 2018. № 18. (Las familias y el derecho de las familias a dos años de vigencia del Código Civil y Comercial) Pgs 62-81 ISNN 1852-2971. Instituto de Cultura Jurídica y Maestría en Sociología Jurídica. FCJ y S. UNLP

\title{
Cuando la realidad supera la norma: gestación por sustitución y filiación post mortem. Los efectos de su omisión
}

Lack of legislation to face new realities, surrogacy and post-mortem filiation bills and the consequences of their elimination

\author{
Marina Ditieri \\ Gilda Beatriz Cortese ${ }^{\bullet \bullet}$ \\ Yanina Graciela González Demaría $\bullet \bullet$
}

\section{Resumen}

El nuevo Código Civil y Comercial de la Nación, edificado sobre la base de la doctrina del derecho internacional de los derechos humanos, se ha presentado como un Código basado en el principio de igualdad y no discriminación, un Código intencionado hacia una mirada pro persona). Sin embargo, la eliminación de dos institutos proyectados -la gestación por sustitución y la filiación post mortem- ha dejado fuera del marco legal la previsión de situaciones que acontecen en la realidad con la desprotección que ello implica para las personas. El presente trabajo tiene como objetivo efectuar un análisis profundizado del modo en que el Anteproyecto de Código Civil y Comercial de la Nación preveía dichos institutos, así como de las consecuencias de su omisión. Finalmente, analizaremos las resoluciones judiciales dictadas con posterioridad a la entrada en vigencia del Código, para así demostrar como la jurisprudencia ha venido a poner en evidencia la necesidad de la sanción de leyes especiales que regulen la materia, volviendo antiguo un código de fondo sancionado hace apenas 2 años.

Palabras claves: Anteproyecto; Código Civil y Comercial de la Nación; Gestación por sustitución; Filiación post mortem; Supresión; Desprotección.

\footnotetext{
Abstract

The new Civil and Commercial Code of Argentina, built on the basis of the opinion of legal experts on international human rights law, has been presented as a Code grounded on the principles of equality and nondiscrimination; in short, a Code with a pro homine view on reality. However, the elimination of two draft bills

- Marina Ditieri, Abogada (UBA) -Maestranda en Derechos Humanos (UNLP) - Docente Universitaria (UBA/UNPAZ/UNDAV) marinaditieri@gmail.com

• Gilda Beatriz Cortese, Abogada, (UBA) - Especialista en Políticas Públicas de Niñez, Adolescencia y Familia (UNER) gildacortese@gmail.com.

•• Yanina Graciela González Demaría, Abogada (UBA) - Especialista en Políticas Públicas de Niñez, Adolescencia y Familia (UNER) gonzalezdemaria@gmail.com

Recibido: 4/3/ 2018 - Aceptado con correcciones: 22/4/2018
} 
Ditieri, M.; Cortese, G.B. y González Demaría, Y. G. Cuando la realidad supera la norma: gestación por sustitución y filiación post mortem. Los efectos de su omisión. Derecho y Ciencias Sociales. Abril 2018. № 18. (Las familias y el derecho de las familias a dos años de vigencia del Código Civil y Comercial) Pgs 62-81 ISNN 1852-2971. Instituto de Cultura Jurídica y Maestría en Sociología Jurídica. FCJ y S. UNLP

-surrogacy and post-mortem filiation- meant excluding from the legal framework the contemplation of actual cases, leaving them without legal protection. The purpose of this paper is to take a critical look at the way in which the Draft Civil and Commercial Code of Argentina provided for such legal devices as well as the consequences of eliminating them. Finally, we will analyse the judicial decisions issued after the Code became effective, in order to demonstrate how precedent has come to show the need for the enactment of special laws regulating that subject-matter, turning old a substantive Code which was sanctioned only two years ago.

Keywords: Draft bill; Civil and Commercial Code of Argentina; Surrogacy; Post-mortem filiation; Elimination; Lack of legal protection. 
Ditieri, M.; Cortese, G.B. y González Demaría, Y. G. Cuando la realidad supera la norma: gestación por sustitución y filiación post mortem. Los efectos de su omisión. Derecho y Ciencias Sociales. Abril 2018. № 18. (Las familias y el derecho de las familias a dos años de vigencia del Código Civil y Comercial) Pgs 62-81 ISNN 1852-2971. Instituto de Cultura Jurídica y Maestría en Sociología Jurídica. FCJ y S. UNLP

\title{
Cuando la realidad supera la norma: gestación por sustitución y filiación post mortem. Los efectos de su omisión
}

\author{
Marina Ditieri \\ Gilda Beatriz Cortese \\ Yanina Graciela González Demaría
}

\section{1.- Introducción.}

El presente artículo tiene como objetivo analizar el modo en que el Proyecto de Código Civil y Comercial de la Nación receptaba en su versión original dos institutos que luego no fueron contemplados: la gestación por sustitución y la filiación post mortem. Puntualmente, efectuaremos una mirada crítica del modo en que los mismos pretendían ser regulados y de los motivos de su omisión. Finalmente, y teniendo en cuenta el dinamismo, complejidad y pluralidad de las relaciones familiares actuales, señalaremos cómo la jurisprudencia ha venido a dar respuesta a la anomia en la materia volviendo antiguo un código de fondo recientemente sancionado.

\section{2.- El Proyecto de Código Civil y Comercial de la Nación.}

En el año 2011, mediante el decreto 191/11, se creó la Comisión Reformadora, integrada por los Dres. Ricardo Luis Lorenzetti, Elena Highton de Nolasco y Aída Kemelmajer de Carlucci, encargada de reformar y asimismo unificar el Código Civil con el Código de Comercio. El Código Civil, por entonces vigente, databa del año 1871 y pese haber sufrido modificaciones parciales, entre las que cuenta con mayor relevancia la producida por la ley 17.711, no lograba aggiornarse a los nuevos escenarios sociales. Por su parte, en el campo del derecho de familia los cambios venían siendo de gran escala y ya no se podía continuar desoyendo las nuevas realidades familiares.

Asimismo, la doctrina del Derecho Internacional de los Derechos Humanos venía avanzando con firmeza hacia otras ramas del derecho. Por tanto, el proceso de constitucionalización del derecho privado, así denominado por la Comisión Redactora, y la incorporación de los tratados de derechos humanos al bloque de constitucionalidad (art. 75 
Ditieri, M.; Cortese, G.B. y González Demaría, Y. G. Cuando la realidad supera la norma: gestación por sustitución y filiación post mortem. Los efectos de su omisión. Derecho y Ciencias Sociales. Abril 2018. № 18. (Las familias y el derecho de las familias a dos años de vigencia del Código Civil y Comercial) Pgs 62-81 ISNN 1852-2971. Instituto de Cultura Jurídica y Maestría en Sociología Jurídica. FCJ y S. UNLP

inc. 22 Constitución Nacional), vinieron a conciliar los principios del derecho público, el derecho privado y la Constitución Nacional. No ajeno a ello, en materia de filiación, el Anteproyecto receptó las mandas convencionales y constitucionales, produciendo un profundo cambio en la materia.

\subsection{La filiación en el Anteproyecto.}

Como hemos señalado, el cambio propuesto en materia filial fue trascendente. A partir de su Título V "Filiación” el anteproyecto (Comisión de Reformas, 2012) receptó entre otras varias cuestiones, modificaciones tendientes a equiparar el sistema filial, teniendo en cuenta la sanción de la Ley 26.618 de Matrimonio Igualitario, y garantizó la consolidación del derecho humano a formar una familia con la incorporación de las técnicas de reproducción humana asistida (en adelante, TRHA) como nueva fuente filial.

Ahora bien, y centrándonos en lo que aquí nos convoca, son dos las temáticas que el Anteproyecto previó y que fueron eliminadas en fecha 20 de noviembre de 2013 mediante un dictamen de la Comisión Bicameral (Honorable Congreso de la Nación, 2012) creada al efecto, y que poseía el proyecto de reforma bajo análisis, obteniendo finalmente media sanción en la Cámara de Senadores el día 28 del mismo mes: la gestación por sustitución (en adelante, GS) y la filiación post mortem (en adelante, FPM).

\section{3.- La gestación por sustitución.}

A modo de introducción, señalamos que la GS es una TRHA por medio de la cual una persona, denominada gestante, sin aportar su material genético (óvulos), lleva adelante un embarazo a partir de la transferencia de un embrión conformado con material genético de los futuros progenitores -comitentes- y/o de terceras personas, donantes de gametos. En uno u otro caso, es decir, con gametos propios de los comitentes o con gametos donados por terceras personas, el niño/a nacido de un procedimiento de GS tiene vínculos jurídicos de filiación con el/los comitente/s (RODRIGUEZ ITURBURU, 2018).

Por su parte, el Anteproyecto preveía la GS con los siguientes alcances:

Artículo 562.- Gestación por sustitución. El consentimiento previo, informado y libre de todas las partes intervinientes en el proceso de gestación por sustitución debe 
Ditieri, M.; Cortese, G.B. y González Demaría, Y. G. Cuando la realidad supera la norma: gestación por sustitución y filiación post mortem. Los efectos de su omisión. Derecho y Ciencias Sociales. Abril 2018. № 18. (Las familias y el derecho de las familias a dos años de vigencia del Código Civil y Comercial) Pgs 62-81 ISNN 1852-2971. Instituto de Cultura Jurídica y Maestría en Sociología Jurídica. FCJ y S. UNLP

ajustarse a lo previsto por este Código y la ley especial. La filiación queda establecida entre el niño nacido y el o los comitentes mediante la prueba del nacimiento, la identidad del o los comitentes y el consentimiento debidamente homologado por autoridad judicial. El juez debe homologar sólo si, además de los requisitos que prevea la ley especial, se acredita que: a) se ha tenido en miras el interés superior del niño que pueda nacer; b) la gestante tiene plena capacidad, buena salud física y psíquica; c) al menos uno de los comitentes ha aportado sus gametos; d) el o los comitentes poseen imposibilidad de concebir o de llevar un embarazo a término; e) la gestante no ha aportado sus gametos; f) la gestante no ha recibido retribución; g) la gestante no se ha sometido a un proceso de gestación por sustitución más de DOS (2) veces; h) la gestante ha dado a luz, al menos, UN (1) hijo propio. Los centros de salud no pueden proceder transferencia embrionaria en la gestante sin la autorización judicial. Si se carece de autorización judicial previa, la filiación se determina por las reglas de la filiación por naturaleza.

En sus Fundamentos se estableció que por aplicación de los principios constitucionales de fundar una familia, el reconocimiento de las diversas formas de organización familiar y el principio de igualdad y no discriminación, el acceso a las técnicas de reproducción humana asistida debía ser admitido de modo amplio. Asimismo, y atento la sanción de la Ley 26.618, se pretendió, como parte de los Fundamentos del Anteproyecto, que el sistema filial esté en plena coincidencia con la nueva conceptualización del matrimonio.

Ahora bien, como antes se señaló, dicha disposición fue eliminada del texto definitivo al pasar por la Cámara de Senadores. El dictamen de la Comisión Bicameral sostuvo que: “... La gestación por sustitución encierra dilemas éticos y jurídicos de gran envergadura que ameritarían un debate más profundo de carácter interdisciplinario. En este contexto de incertidumbre y cuasi silencio legal en el Derecho Comparado, se propone de manera precautoria, eliminar la gestación por sustitución del Proyecto de reforma" (Honorable Congreso de la Nación, 2012).

Como puede observarse, los motivos de la eliminación respondieron a planteos éticos y jurídico pero como era de prever desconociéndose la realidad, ya que incluso antes de la la GS se llevaba a cabo mediante autorización judicial. Y aquí debemos preguntarnos: ¿Qué 
Ditieri, M.; Cortese, G.B. y González Demaría, Y. G. Cuando la realidad supera la norma: gestación por sustitución y filiación post mortem. Los efectos de su omisión. Derecho y Ciencias Sociales. Abril 2018. № 18. (Las familias y el derecho de las familias a dos años de vigencia del Código Civil y Comercial) Pgs 62-81 ISNN 1852-2971. Instituto de Cultura Jurídica y Maestría en Sociología Jurídica. FCJ y S. UNLP

ha cambiado para que no continúe llevándose adelante? Asimismo, ¿Su no regulación llevaría implícita su prohibición?

Dichos cuestionamientos tuvieron una pronta respuesta desde la academia, poco después de la entrada en vigencia del Código Civil y Comercial (en adelante, $\mathrm{CCyCN}$ ), a partir de las conclusiones arribadas en las Jornadas Nacionales de Derecho Civil celebradas en Bahía Blanca en octubre del año 2015. Allí la Comisión $N^{\circ} 6$ sobre Familia: “Identidad y filiación” resolvió por unanimidad que: “Aún sin ley, al no estar prohibida, se entiende que la gestación por sustitución está permitida”. Por tanto, pese a la supresión del texto del nuevo Código, la gestación por sustitución no fue prohibida. Ante ello, continúa practicándose y los casos hoy planteados son resueltos en sede judicial.

En este punto, es importante hacer un breve análisis del primer caso que se presentó ante la justicia, incluso con anterioridad a la sanción del nuevo $\mathrm{CCyCN}$, en fecha 18/06/2013, por inscripción de nacimiento. En dicho caso, un matrimonio heterosexual apela a la gestación por sustitución recurriendo a la ayuda de una amiga que se ofrece de manera altruista a gestar el embrión compuesto por el material genético de ambos cónyuges, debido a que la comitente se veía imposibilitada de gestar (no solo por haber perdido otros embarazos sino porque además se le debió practicar una intervención quirúrgica que incluyó la extirpación del útero). La gestante era madre de dos hijos mayores de edad y vivía en unión convivencial desde hacía ocho años. Todos ellos estaban informados sobre su voluntad de ayudar al matrimonio con el fin de llevar adelante el embarazo. Consecuentemente, cuando la niña gestada nace, se expide un certificado de nacimiento que tiene como madre a la gestante en consonancia con lo prescripto en el art. 242 del Código Civil.

Frente a ello, el matrimonio decide no inscribir en el registro el nacimiento e interpone una acción judicial solicitando la inscripción de la niña como hija del matrimonio. La jueza actuante hizo lugar a la demanda y ordenó al Registro del Estado Civil y Capacidad de las Personas de la Ciudad de Buenos Aires a que efectúe la inscripción del nacimiento de la niña nacida el 19/04/2012 como hija del matrimonio integrado por los comitentes, tomando como argumento central de su decisión: la falta de regulación de la gestación por sustitución y la voluntad procreacional, definiéndola como 
Ditieri, M.; Cortese, G.B. y González Demaría, Y. G. Cuando la realidad supera la norma: gestación por sustitución y filiación post mortem. Los efectos de su omisión. Derecho y Ciencias Sociales. Abril 2018. № 18. (Las familias y el derecho de las familias a dos años de vigencia del Código Civil y Comercial) Pgs 62-81 ISNN 1852-2971. Instituto de Cultura Jurídica y Maestría en Sociología Jurídica. FCJ y S. UNLP

"la intención de querer engendrar un hijo con material biológico propio, empero, acudiendo a la portación del embrión en el vientre de un tercero para su gestación y alumbramiento posterior" 1 .

Ya en plena vigencia del nuevo CCyCN con fecha 30/12/2015, se dicta otro de los precedentes significativos en la Provincia de Buenos Aires ante el Tribunal de Familia no 3 de Lomas de Zamora a cargo de la Dra. María Silvia Villaverde. En este caso, una pareja heterosexual, imposibilitada de procrear (debido a que a la mujer le fue diagnosticada una enfermedad congénita que le impedía gestar, pero no producir óvulos) aportó sus gametos para la gestación de su hija, respecto de la cual habían expresado su voluntad procreacional mediante el consentimiento previo, libre e informado. La hermana de la mujer se ofreció como gestante, quien al momento de la práctica era casada y tenía tres hijos menores de edad y todos conocían su decisión. Así las cosas, se requirió la inscripción del nacimiento de la niña como hija de los integrantes de la pareja que había expresado su voluntad procreacional en el consentimiento informado, y no como hija de la mujer gestante.

La jueza resolvió haciendo lugar a la gestación por sustitución ordenando su inscripción inmediata como hija de los comitentes, declarando "la inconstitucionalidad y anticonvencionalidad del art. 562 del Código Civil y Comercial de la Nación en cuanto no reconoce la maternidad de la mujer que ha expresado su voluntad procreacional mediante el consentimiento informado, sino la de la mujer que da a luz". De esta forma la jueza entendió que la gestación por sustitución “contaría con recepción implícita en el CCCN, por considerar que la falta de mención expresa de este tipo de TRHA no implica prohibición”.

Asimismo, opinó que

la voluntad procreacional es querer engendrar un hijo, darle afecto y asumir la responsabilidad de su formación integral, en el marco del derecho a una maternidad y a una paternidad libre y responsable, sin exclusiones irrazonables y respetando la diversidad como característica propia de la condición humana y de la familia, y se expresa mediante el otorgamiento del consentimiento previo, libre e informado". Concluyó que: "La gestación por sustitución, de acuerdo a la diversidad funcional de la progenitora, constituye para esta pareja la única oportunidad real de ejercer su derecho

\footnotetext{
${ }^{1}$ Fallo que se puede consultar en la Revista Jurídica La Ley. Buenos Aires. La Ley, tomo D, pag. 195.
} 
Ditieri, M.; Cortese, G.B. y González Demaría, Y. G. Cuando la realidad supera la norma: gestación por sustitución y filiación post mortem. Los efectos de su omisión. Derecho y Ciencias Sociales. Abril 2018. № 18. (Las familias y el derecho de las familias a dos años de vigencia del Código Civil y Comercial) Pgs 62-81 ISNN 1852-2971. Instituto de Cultura Jurídica y Maestría en Sociología Jurídica. FCJ y S. UNLP

fundamental a formar una familia y de ejercer una maternidad y una paternidad responsable y en igualdad de condiciones que los demás. (Fallo del Juzgado de Familia Nro. 7 de Lomas de Zamora “ B.J.D y otros s/Materia a categorizar)

Posteriormente, la misma magistrada, en el caso de caso de GS, el fallo del Juzgado Nro. 7 de Familia de Lomas de Zamora en autos “ B.J.D y otros s/ materia a categorizar” declara nuevamente, la inconstitucionalidad y anticonvencionalidad del art. 562 del CCyCN al reconocer la gestación por sustitución de una abuela que dio a luz a su nieto, inscribiéndose inmediatamente el nacimiento del niño como hijo de la pareja matrimonial que había expresado su voluntad procreacional mediante los correspondientes consentimientos informados.

Con el objeto de contrarrestar el silencio del nuevo $\mathrm{CCyCN}$ y con el fin de evitar que las personas dependan del criterio judicial para el caso en concreto, ya se han presentado varios proyectos de $1 \mathrm{ey}^{2}$, con el fin de legislar y regular la GS. El último de ellos ${ }^{3}$, presentado ante la Cámara de Diputados de la Nación en el mes de marzo del 2018, da cuenta de las 23 resoluciones judiciales recaídas a favor del uso de la técnica. A continuación, y tal como lo remarca dicho proyecto, se señalan los casos en cuestión:

\begin{tabular}{|c|c|c|}
\hline JUZGADO & FECHA & AUTOS \\
\hline $\begin{array}{l}\text { 1) Juzgado de Familia de } \\
\text { Gualeguay }\end{array}$ & 19 de noviembre de 2013 & "B., M. A. v. F. C., C. R. - ordinario \\
\hline $\begin{array}{l}\text { 2) Juzgado Nacional en lo Civil } \mathrm{N}^{\circ} \\
86\end{array}$ & 18 de junio de 2013 & $\begin{array}{l}\text { "N. N. o DGMB s/inscripción de } \\
\text { nacimiento" }\end{array}$ \\
\hline $\begin{array}{l}\text { 3) Tribunal de Familia de Rosario } \\
\mathrm{N}^{\circ} 7\end{array}$ & 2 de diciembre de 2014 & $\begin{array}{l}\text { "F. M. L. y otra s/Autorización } \\
\text { judicial" }\end{array}$ \\
\hline $\begin{array}{l}\text { 4) Juzgado Nacional en lo Civil } \mathrm{N}^{\circ} \\
102\end{array}$ & 18 de mayo de 2015 & $\begin{array}{l}\text { "C., F. A. y otro c/R. S., M. L. } \\
\text { s/Impugnación de maternidad" }\end{array}$ \\
\hline $\begin{array}{l}\text { 5) Juzgado Nacional en lo Civil } \mathrm{N}^{\circ} \\
83\end{array}$ & 30 de junio de 2015 & $\begin{array}{l}\text { "N. N. O. s/Inscripción de } \\
\text { nacimiento" }\end{array}$ \\
\hline
\end{tabular}

\footnotetext{
${ }^{2}$ Proyecto 5700-D-2016. Proyecto 5759-D-2016. Proyecto 3202-D-2017. Proyecto 3765-D-2017. Proyecto 5141-D-2017.

${ }^{3}$ Proyecto 0084-D-2018.
} 
Ditieri, M.; Cortese, G.B. y González Demaría, Y. G. Cuando la realidad supera la norma: gestación por sustitución y filiación post mortem. Los efectos de su omisión. Derecho y Ciencias Sociales. Abril 2018. № 18. (Las familias y el derecho de las familias a dos años de vigencia del Código Civil y Comercial) Pgs 62-81 ISNN 1852-2971. Instituto de Cultura Jurídica y Maestría en Sociología Jurídica. FCJ y S. UNLP

\begin{tabular}{|c|c|c|}
\hline $\begin{array}{l}\text { 6) Juzgado de Familia } \mathrm{N}^{\circ} 1 \mathrm{de} \\
\text { Mendoza }\end{array}$ & 29 de julio de 2015 & $\begin{array}{l}\text { "O. A. V. p/Medida } \\
\text { Autosatisfactiva" }\end{array}$ \\
\hline $\begin{array}{l}\text { 7) Juzgado de Familia } N^{\circ} 1 \text { de } \\
\text { Mendoza }\end{array}$ & 15 de diciembre de 2015 & $\begin{array}{l}\text { "C. M. E. y J. R. M. por inscripción } \\
\text { de nacimiento" }\end{array}$ \\
\hline $\begin{array}{l}\text { 8) Juzgado de Familia } \mathrm{N}^{\circ} 9 \text { de San } \\
\text { Carlos de Bariloche }\end{array}$ & 29 de diciembre de 2015 & $\begin{array}{l}\text { "Dato reservado Expte. } \mathrm{N}^{\circ} 10.178 \\
14 "\end{array}$ \\
\hline $\begin{array}{l}\text { 9) Juzgado de Familia } \mathrm{N}^{\circ} 7 \mathrm{de} \\
\text { Lomas de Zamora }\end{array}$ & 30 de diciembre de 2015 & $\begin{array}{l}\text { "H. M. y otro/a s/Medidas } \\
\text { precautorias" }\end{array}$ \\
\hline $\begin{array}{l}\text { 10) Tribunal Colegiado de Familia } \\
\mathrm{N}^{\circ} 5 \text { de Rosario }\end{array}$ & 27 de mayo de 2016 & "S. G. G. y otros s/Filiación" \\
\hline $\begin{array}{l}\text { 11) Juzgado Nacional en lo Civil N } \\
{ }^{\circ} 7\end{array}$ & 23 de mayo de 2016 & $\begin{array}{l}\text { “A. R., C y otros c/ C., M. J. } \\
\text { s/Impugnación de filiación”" }\end{array}$ \\
\hline $\begin{array}{l}\text { 12) Juzgado Nacional en lo Civil N } \\
{ }^{\circ} 4\end{array}$ & 30 de junio de 2016 & $\begin{array}{l}\text { "S. T., A y otro s/Inscripciòn de } \\
\text { nacimiento" (sentencia no firme) }\end{array}$ \\
\hline 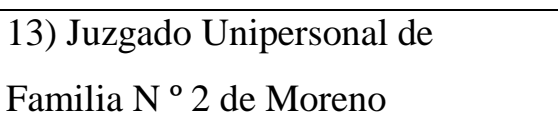 & 4 de julio de 2016 & $\begin{array}{l}\text { "“S. P., B. B. c/S. P., R. F. s/materia } \\
\text { a categorizar" }\end{array}$ \\
\hline $\begin{array}{l}\text { 14) Juzgado Nacional en lo Civil N } \\
{ }^{\circ} 8\end{array}$ & 20 de septiembre de 2016 & $\begin{array}{l}\text { "B., B. M. y otro c/ G., Y. A. } \\
\text { s/impugnación de filiación" }\end{array}$ \\
\hline $\begin{array}{l}\text { 15) Juzgado de Familia } N^{\circ} 3 \text { de } \\
\text { Gral. San Martín }\end{array}$ & 22 de agosto de 2016 & $\begin{array}{l}\text { "M., I. M. y otro s/autorización } \\
\text { judicial" }\end{array}$ \\
\hline $\begin{array}{l}\text { 16) Juzgado de Familia } \mathrm{N}^{\circ} 12 \text { de } \\
\text { Lomas de Zamora }\end{array}$ & 3 de octubre de 2016 & $\begin{array}{l}\text { "G. M., C y otro c/ W. B., A. V } \\
\text { s/rectificación de partida" }\end{array}$ \\
\hline $\begin{array}{l}\text { 17) Juzgado de Familia } \mathrm{N}^{\circ} 7 \mathrm{de} \\
\text { Lomas de Zamora }\end{array}$ & 30 de noviembre de 2016 & $\begin{array}{l}\text { "B. J. D. y Otros s/ materia a } \\
\text { categorizar" }\end{array}$ \\
\hline $\begin{array}{l}\text { 18) Juzgado Nacional en lo Civil } \\
\mathrm{N}^{\circ} 81\end{array}$ & 14 de junio de 2017 & $\begin{array}{l}\text { "S., I. N. y otro c/A., C. L. } \\
\text { s/Impugnación de Filiación" }\end{array}$ \\
\hline $\begin{array}{l}\text { 19) Juzgado de Familia } \mathrm{N}^{\circ} 7 \mathrm{de} \\
\text { Viedma }\end{array}$ & 6 de julio de 2017 & "Reservado s/ Autorización Judicial" \\
\hline $\begin{array}{l}\text { 20) Juzgado de Familia } \mathrm{N}^{\circ} 1 \text { de } \\
\text { Mendoza }\end{array}$ & 6 de septiembre de 2017 & $\begin{array}{l}\text { "M.M.C. y M.G.J. y R.F.N. por } \\
\text { Medidas Autosatisfacías" }\end{array}$ \\
\hline $\begin{array}{l}\text { 21) Juzgado Nacional en lo Civil } \\
\mathrm{N}^{\circ} 4\end{array}$ & 20 de octubre de 2017 & $\begin{array}{l}\text { "S. T., V. } \text { s/inscripción } \mathrm{de} \\
\text { nacimiento" (sentencia no firme). }\end{array}$ \\
\hline
\end{tabular}


Ditieri, M.; Cortese, G.B. y González Demaría, Y. G. Cuando la realidad supera la norma: gestación por sustitución y filiación post mortem. Los efectos de su omisión. Derecho y Ciencias Sociales. Abril 2018. № 18. (Las familias y el derecho de las familias a dos años de vigencia del Código Civil y Comercial) Pgs 62-81 ISNN 1852-2971. Instituto de Cultura Jurídica y Maestría en Sociología Jurídica. FCJ y S. UNLP

\begin{tabular}{|l|l|l|}
\hline $\begin{array}{l}\text { 22) Juzgado de Familia N } \mathrm{N}^{\mathrm{a}} 3 \\
\text { Córdoba }\end{array}$ & 22 de noviembre de 2017 & $\begin{array}{l}\text { "R., L. S. y otros - solicita } \\
\text { homologacion” (expte. } \mathrm{n}^{\text {o } 3447358) .}\end{array}$ \\
\hline $\begin{array}{l}\text { 23) Tribunal Colegiado de Familia } \\
\mathrm{N}^{\mathrm{a}} 7 \text { Rosario }\end{array}$ & 5 de diciembre de 2017 & $\begin{array}{l}\text { "H., M.E. y otros s/ venias y } \\
\text { dispensas". }\end{array}$ \\
\hline
\end{tabular}

Del análisis de dichos precedentes surgen particularidades, a saber: En los casos $\mathrm{N}^{\circ}$ 9 y $\mathrm{N}^{\circ} 12$ la persona gestante era familiar de los comitentes. En el caso $\mathrm{N}^{\circ} 10$ el embrión transferido a la persona gestante se conformó, parcialmente, con material genético del matrimonio comitente, en tanto los gametos masculinos los aportó el cónyuge comitente y los gametos femeninos una persona ovo donante. En los casos $\mathrm{N}^{\circ} 11$ y $\mathrm{N}^{\circ} 13$ la GS se reconoció a favor de parejas de dos hombres. En los casos $\mathrm{N}^{\circ} 9$ y $\mathrm{N}^{\circ} 13$ se declaró la inconstitucionalidad del artículo 562 del CCyC actualmente vigente. En los casos $\mathrm{N}^{\circ} 3,8$, 15, 19, 22 y 23 el/los comitentes requirieron autorización judicial previa a la realización del procedimiento de la $\mathrm{GS}^{4}$.

Asimismo, en el campo del Derecho Comparado, son muchos los ordenamientos que están regulando la GS para dar respuesta y solución a una práctica cada vez más frecuente. El número de Estados que tienen leyes que regulan la GS está creciendo en estos últimos diez años: Australia (Act 2004): Queensland (2010), New South Wales (2010), Western Australia (2008), Victoria (2008), Canadá: Alberta (2010), Columbia Británica (2011, aún no en vigor), Grecia (2002 y 2005), Rusia (2011), Sudáfrica (2010), Uruguay (2013). Asimismo, algunos Estados se están cuestionando su regulación. Tal es el caso de Bélgica, Bulgaria, Finlandia, Islandia, Irlanda, entre otros. Muchas legislaciones que eran mayormente restrictivas, se están flexibilizando, tal es el caso de Rusia, Reino Unido, Grecia, Israel, Brasil y España (HERRERA - LAMM, 2014). También son conocidos los países donde la práctica está permitida y usualmente se realiza lo que suele llamarse "turismo reproductivo", tales son países como Ucrania, India y algunos estados de los Estados Unidos.

Por su parte, el Tribunal Europeo de Derechos Humanos (TEDH) con fecha 26/06/2014 ha tenido la oportunidad de expedirse a favor de la gestación por sustitución

\footnotetext{
${ }^{4}$ Para ampliar véase proyecto 0084-D-2018.
} 
Ditieri, M.; Cortese, G.B. y González Demaría, Y. G. Cuando la realidad supera la norma: gestación por sustitución y filiación post mortem. Los efectos de su omisión. Derecho y Ciencias Sociales. Abril 2018. № 18. (Las familias y el derecho de las familias a dos años de vigencia del Código Civil y Comercial) Pgs 62-81 ISNN 1852-2971. Instituto de Cultura Jurídica y Maestría en Sociología Jurídica. FCJ y S. UNLP

ante dos casos similares tramitados en Francia, estos son: "Mennesson" y "Labassee". En el primero de ellos, el matrimonio francés celebra en California en el año 2000 un contrato de gestación por sustitución con una norteamericana por el cual la misma dará a luz al futuro hijo de la pareja, concebido con los gametos del padre y un óvulo donado. La Corte Suprema de dicho Estado, con fecha 14/7/2000, confiere la calidad de padres a los esposos franceses y ordena la inscripción de las dos niñas nacidas como hijas del matrimonio en las respectivas partidas.

Ante ello, el progenitor solicita al consulado de su país en Los Ángeles la transcripción de dichas actas, pedido que le es denegado en tanto se infiere que las niñas habían nacido por medio de una gestación por sustitución. Una vez arribado el grupo familiar a Francia, el matrimonio sin aportar pruebas del nacimiento, peticiona que las actas le sean transcriptas en los registros de estado civil de Nantes. Así las cosas, el Ministerio Fiscal pide su anulación basándose en razones de orden público. El 25/10/2007, la Corte de Apelación de París rechaza la acción de nulidad interpuesta por el Ministerio Fiscal. Se invoca, entre otras cosas, el reiterado interés superior de las niñas, siendo finalmente dicha decisión recurrida ante la Corte de Casación. En fecha 18/3/2010, esta última instancia consideró que la transcripción de las actas de nacimiento viola el orden público francés, por lo que se ordenó su anulación. Frente a esta resolución, el matrimonio recurre ante el TEDH, aduciendo que

la decisión que reconoce la filiación de un niño nacido por gestación por sustitución como hijo de una pareja no es contraria al orden público internacional francés. Afirman que debe tenerse en cuenta el interés de estas niñas que no solo existen, sino que han forjado vínculos afectivos fuertes y sólidos con el matrimonio, por lo cual lo decidido implicaría privar a los niños de la posibilidad de establecer su filiación en Francia, donde ya tienen su residencia y de este modo, violar el art. 8 de la Convención Europea de los Derechos del Hombre (CEDH), que regula el respeto a la vida familiar y privada como así el art. 14, de no discriminación, al tratarlos de manera diferente a la de otros infantes, solo por haber nacido por gestación por sustitución, situación totalmente ajena a la decisión de los niños y por la cual son los principales perjudicados.

\footnotetext{
${ }^{5}$ Para ampliar véase La Ley Online. Cita online AR/DOC/2285/2014
} 
Ditieri, M.; Cortese, G.B. y González Demaría, Y. G. Cuando la realidad supera la norma: gestación por sustitución y filiación post mortem. Los efectos de su omisión. Derecho y Ciencias Sociales. Abril 2018. № 18. (Las familias y el derecho de las familias a dos años de vigencia del Código Civil y Comercial) Pgs 62-81 ISNN 1852-2971. Instituto de Cultura Jurídica y Maestría en Sociología Jurídica. FCJ y S. UNLP

En ambos casos, con argumentos similares, y por unanimidad el Tribunal ha dicho que si bien no se encuentra violado el art. 8 de la Convención Europea de Derechos Humanos (Derecho a la vida privada) con relación a los demandantes, sí hubo tal violación con relación al derecho de los niños involucrados a que se les respete tal derecho. El TEDH consideró que las medidas adoptadas por el Estado Francés fueron contrarias al interés superior de los niños perjudicados en tanto el respeto de la vida privada se vincula con la esencia de la identidad incluyendo la filiación.

Una vez más, al momento de resolver, y en esta ocasión un tribunal especializado en materia de derechos humanos, se pronunció a favor de la gestación por sustitución, ponderando la verdad consentida por encima de la genética.

Por último, resulta relevante señalar que en el ámbito del derecho internacional privado de nuestro país, a través del art. 2634 del $\mathrm{CCyCN}$, se reconoce el emplazamiento filial de los casos de GS llevadas a cabo en el extranjero ${ }^{6}$.

\section{4.- La filiación post mortem.}

La llamada filiación post mortem es una especie de TRHA que se lleva a cabo luego de la muerte de uno de los miembros de la pareja conviviente o del matrimonio, con las particularidades que a continuación se irán detallando. Al igual que la GS, la FPM pretendió ser incorporada en el Anteproyecto del CCyCN mediante el art. 563. Dicho artículo establecía:

En caso de muerte del o la cónyuge o conviviente de la mujer que da a luz, no hay vínculo filial entre la persona nacida del uso de las técnicas de reproducción humana asistida y la persona fallecida si la concepción en la mujer o la implantación del embrión en ella no se había producido antes del fallecimiento. No rige lo dispuesto en el párrafo anterior si se cumple con los siguientes requisitos: a) la persona consiente en

\footnotetext{
${ }^{6}$ Articulo 2634 CCyCN: Reconocimiento de emplazamiento filial constituido en el extranjero. Todo emplazamiento filial constituido de acuerdo con el derecho extranjero debe ser reconocido en la República de conformidad con los principios de orden público argentino, especialmente aquellos que imponen considerar prioritariamente el interés superior del niño.

Los principios que regulan las normas sobre filiación por técnicas de reproducción humana asistida integran el orden público y deben ser ponderados por la autoridad competente en ocasión de que se requiera su intervención a los efectos del reconocimiento de estado o inscripción de personas nacidas a través de estas técnicas. En todo caso, se debe adoptar la decisión que redunde en beneficio del interés superior del niño.
} 
Ditieri, M.; Cortese, G.B. y González Demaría, Y. G. Cuando la realidad supera la norma: gestación por sustitución y filiación post mortem. Los efectos de su omisión. Derecho y Ciencias Sociales. Abril 2018. № 18. (Las familias y el derecho de las familias a dos años de vigencia del Código Civil y Comercial) Pgs 62-81 ISNN 1852-2971. Instituto de Cultura Jurídica y Maestría en Sociología Jurídica. FCJ y S. UNLP

el documento previsto en el Art. 560 o en un testamento que los embriones producidos con sus gametos sean transferidos en la mujer después de su fallecimiento, b) la concepción en la mujer o la implantación del embrión en ella se produce dentro del año siguiente al deceso.

De este modo, el Anteproyecto contemplaba la FPM, siendo la regla su prohibición, con las excepciones mencionadas en la parte final del artículo en cuestión. Cabe aclarar, que existen tres posiciones frente a la FPM las cuales son: su prohibición expresa, su regulación o el silencio legislativo. En este caso, el Anteproyecto se inclinó por la regulación de la FPM brindando seguridad jurídica a las partes intervinientes. Sin embargo, sin mayores fundamentos se eliminó su regulación y se desdobló parte del texto originario del artículo 563 de manera que el primer párrafo pasó a ser el nuevo artículo 562 "voluntad procreacional" y el segundo párrafo quedó como el nuevo Art. 563 “derecho a la información de las personas nacidas por técnicas de reproducción humana asistida" (MERLO, 2013), no logrando arribar a la inclusión de la FPM en el CCyCN vigente.

Para agregar, señalaremos que hubo voces que se pronunciaron expresamente en contra de la FPM afirmando que, de permitirse este tipo de regulación, se estaría condenando a un niño deliberada y conscientemente a nacer huérfano privilegiando, de esta manera, el interés del progenitor y no del niño. Se suma a esta postura que en la FPM se coloca al hijo en forma voluntaria en un hogar disgregado, menoscabando el principio del interés superior del niño, constituyendo la FPM un ejercicio abusivo y desmesurado del derecho a procrear. De llevarse a cabo esta modalidad, se puede afectar el desarrollo y la personalidad del niño atento a la carencia de un progenitor y de su relación con el otro resultando indiscutible el derecho de los hijos de ser concebidos, traídos al mundo y educados por ambos padres. Además, se pondría en juego el derecho de la identidad del niño puesto que de no cumplirse con los requisitos establecidos en el artículo 563 del Anteproyecto, no se le podría reconocer al mismo vínculo filial alguno con la persona fallecida ${ }^{7}$.

\footnotetext{
${ }^{7}$ Véase como Anexo I y Anexo II en Http://www.senado.gov.ar/parlamentario/parlamentaria/ordenDelDiaResultadoLink/2013/892, recuperado el 20 de abril de 2018.
} 
Ditieri, M.; Cortese, G.B. y González Demaría, Y. G. Cuando la realidad supera la norma: gestación por sustitución y filiación post mortem. Los efectos de su omisión. Derecho y Ciencias Sociales. Abril 2018. № 18. (Las familias y el derecho de las familias a dos años de vigencia del Código Civil y Comercial) Pgs 62-81 ISNN 1852-2971. Instituto de Cultura Jurídica y Maestría en Sociología Jurídica. FCJ y S. UNLP

Así vemos como uno de los poderes del estado, como lo es el Poder Legislativo se pronunció en materia de FPM, a contrario de lo que fue ocurriendo en el Poder Judicial. Debido al mandato normativo que dispone que los magistrados no pueden dejar de fallar bajo el pretexto de silencio, oscuridad o insuficiencia de las leyes y que los mismos pueden acudir a principios de leyes análogas o principios del derecho atendiendo a las circunstancias del caso para cumplir con su obligación de fallar, la jurisprudencia nacional previa y posterior a la sanción del $\mathrm{CCyCN}$ fue dictaminando a favor de la FPM con diferentes matices viniendo a subsanar el silencio normativo que impera hasta el día de la fecha.

Podemos mencionar como primer antecedente internacional de FPM, el ocurrido en Francia en el año 1984, cuando la viuda Corinne Parpalaix, obtiene la autorización judicial para inseminarse con semen de su marido premuerto. Asimismo, también en Gran Bretaña se dio el caso más conocido como "Blood", en el que el semen extraído se realizó sobre el hombre que estaba en coma. En este caso, Diana Blood, la viuda del hombre que estuvo en coma, obtuvo la autorización para ser inseminada luego de dos años de la extracción y fuera del territorio de Gran Bretaña en el año 1997 (PEREZ, 2014).

Más allá de los antecedentes internacionales que precedieron a las situaciones de FPM en la República Argentina, el primer caso en nuestro país tiene lugar en el año 1999. Se trató de una mujer española que estaba con su pareja de luna de miel cuando el mismo fallece. La mujer solicitó autorización judicial a través del consulado Español para realizar la extracción de material genético de su pareja fallecida y poder llevar a cabo en España una práctica de TRHA. Dicha autorización es finalmente concedida ${ }^{8}$. Posteriormente, se sucedieron una serie de pedidos judiciales de autorización para la realización de la FPM, de los cuales, tres casos son anteriores a la entrada en vigencia del $\mathrm{CCyCN}$ y los dos casos siguientes, son posteriores a la misma.

Ante el silencio normativo y los pedidos de autorización judicial que se fueron otorgando, la jurisprudencia argentina fue resolviendo de manera clara a favor de la FPM. En este sentido, los jueces intervinientes se han expedido sobre diferentes tipos de modalidades de la FPM como la utilización de los gametos crioconservados de la persona

\footnotetext{
${ }^{8}$ Véase https://www.pagina12.com.ar/1999/99-11/99-11-03/pag21.htm, recuperado el 20 de abril de 2018.
} 
Ditieri, M.; Cortese, G.B. y González Demaría, Y. G. Cuando la realidad supera la norma: gestación por sustitución y filiación post mortem. Los efectos de su omisión. Derecho y Ciencias Sociales. Abril 2018. N 18. (Las familias y el derecho de las familias a dos años de vigencia del Código Civil y Comercial) Pgs 62-81 ISNN 1852-2971. Instituto de Cultura Jurídica y Maestría en Sociología Jurídica. FCJ y S. UNLP

fallecida ${ }^{9}$, la utilización de los embriones criopreservados de la pareja ${ }^{10}$ y la extracción compulsiva de material genético de la persona fallecida y posterior tratamiento de TRHA ${ }^{11}$. Los magistrados han esgrimido diferentes argumentos para autorizar la FPM. Incluso, en el caso "S.M.C. s/medida autosatisfactiva" que llegó hasta la Cámara de Apelaciones en lo Civil, Comercial, Minas, de Paz y Tributario de Mendoza por el cual los jueces terminaron revocando la sentencia del tribunal de primera instancia que había denegado la autorización requerida.

En la mayoría de los casos nacionales, los fundamentos en que se apoyan las sentencias judiciales sobre la consecución de la FPM apelan al principio de legalidad del art. 19 de la Constitución Nacional ${ }^{12}$ en cuanto a que ningún habitante de la Nación será obligado a hacer lo que no manda la ley, ni privado de lo que ella no prohíbe. También, se apela a la Ley 26.862 de "Reproducción médicamente asistida" cuyo objeto es el de garantizar el acceso integral a los procedimientos y técnicas médico-asistenciales de reproducción médicamente asistida (Art.1). Esto es concordante con la aplicación de la doctrina de la Corte Interamericana de Derechos Humanos (CIDH) en el caso "Artavia Murillo y otros vs. Costa Rica” que señala el derecho a tener hijos biológicos a través del acceso de las TRHA, que a su vez, se deriva del derecho a la integridad personal, libertad personal, vida privada y familiar. Asimismo, se apela al derecho a la salud reproductiva, derecho a la autonomía de la persona humana, derechos de toda persona a la paternidad/maternidad y a formar una familia, en íntima conexión con el derecho a la salud.

De igual forma, frente al argumento de la creación de niños huérfanos, la mayoría de los magistrados han hecho referencia al caso "Fornerón Vs Argentina" de la CIDH, en

\footnotetext{
${ }^{9}$ Tribunal de Familia de Morón $\mathrm{n}^{\mathrm{o}}$ 3, “G.A.P. s/autorización”, 21/11/11. RDF 2012-III-119, AP/JUR/289/2011; Juzgado Nacional de Primera Instancia en lo Civil $n^{\circ} 3$, “K., J. V. c/Instituto de Ginecología y Fertilidad y otros s/amparo", 03/11/14. La Ley. AR/JUR/53958/2014.

${ }^{10}$ Juzgado de Primera Instancia en lo Civil, Comercial, Laboral y de Minería n 4 de Santa Rosa, La Pampa, “A., C. V. c/Instituto de Seguridad Social-SEMPRE s/amparo", 30/12/2015. DFyP 2016 (agosto). AR/JUR/87457/2015.

${ }^{11}$ Tercera Cámara de Apelaciones en lo Civil, Comercial, Minas, de Paz y Tributario de Mendoza, en Mendoza, "S.M.C. s/medida autosatisfactiva", 07/08/2014. RC J 6303/14. Juzgado Nacional de Primera Instancia en lo Civil no 87, "N.O.C.P. s/autorización", 05/05/2016. La Ley 2016-D 219. AR/JUR/32492/2016.

12 Artículo 19.- Las acciones privadas de los hombres que de ningún modo ofendan al orden y a la moral pública, ni perjudiquen a un tercero, están sólo reservadas a Dios, y exentas de la autoridad de los magistrados. Ningún habitante de la Nación será obligado a hacer lo que no manda la ley, ni privado de lo que ella no prohíbe.
} 
Ditieri, M.; Cortese, G.B. y González Demaría, Y. G. Cuando la realidad supera la norma: gestación por sustitución y filiación post mortem. Los efectos de su omisión. Derecho y Ciencias Sociales. Abril 2018. № 18. (Las familias y el derecho de las familias a dos años de vigencia del Código Civil y Comercial) Pgs 62-81 ISNN 1852-2971. Instituto de Cultura Jurídica y Maestría en Sociología Jurídica. FCJ y S. UNLP

cuanto a la protección de familia monoparental, reconociendo la diversidad de familias. Respecto a que el principio del interés superior del niño se estaría vulnerando, en el caso "K., J. V. c/Instituto de Ginecología y Fertilidad y otros s/amparo", el juez interviniente señaló que aún no hay niño no teniendo certezas si la formación de un embrión y el posterior nacimiento resultará o no exitosa. En el mismo fallo, se recoge el "consentimiento presunto", a partir del consentimiento brindado por la persona para la extracción de sus gametos en vida.

Vemos cómo a casi dos años de la sanción del $\mathrm{CCyCN}$, la jurisprudencia falló con amplitud de criterio y se extendió a supuestos que ni siquiera estaban contemplados en el Anteproyecto.

En primer lugar, el Anteproyecto solo establecía para la FPM, la transferencia de embriones, el consentimiento previo, libre e informado de la persona fallecida y el establecimiento de un plazo de un año para la concepción y/o implantación del embrión en la mujer. La jurisprudencia ha fallado no solo en materia de embriones crioconservados, sino también de material genético crioconservado y de extracción de semen del cuerpo del fallecido.

En segundo lugar, el Poder Judicial ha resuelto, en casos de FPM, con amplitud de plazos, tal es el fallo "N.O.C.P. s/autorización” que tramitó ante el Juzgado Nacional de Primera Instancia en lo Civil nº 87, cuyo pedido de autorización del tratamiento se efectuó cuatro años y medio después del fallecimiento de la persona.

Por último, es interesante puntuar que de haberse aprobado el artículo 563 del Anteproyecto del CCyCN, la FPM se hubiera podido llevar adelante solamente en relación a los "embriones" dejando de lado a los gametos; evidenciando la incoherencia del artículo con la decisión de la CIDH en el caso “Artavia Murillo y otros Vs. Costa Rica” en relación a la noción de que el embrión no es persona. Por consiguiente, si el embrión no es persona no se explica porqué el artículo mencionado excluyó del tratamiento jurídico a los gametos (HERRERA, 2016).

\section{Palabras finales.}


Ditieri, M.; Cortese, G.B. y González Demaría, Y. G. Cuando la realidad supera la norma: gestación por sustitución y filiación post mortem. Los efectos de su omisión. Derecho y Ciencias Sociales. Abril 2018. № 18. (Las familias y el derecho de las familias a dos años de vigencia del Código Civil y Comercial) Pgs 62-81 ISNN 1852-2971. Instituto de Cultura Jurídica y Maestría en Sociología Jurídica. FCJ y S. UNLP

Del análisis conjunto de ambos institutos no regulados, se desprende la imperiosa necesidad de contar con un marco legal que los contemple y que tenga como principal objetivo la salvaguarda de los derechos de los sujetos intervinientes.

En el caso de la GS, si bien, la propuesta efectuada oportunamente por el Anteproyecto resultaba adecuada e incluso novedosa, podríamos decir que su eliminación no es coincidente con los nuevos modelos familiares que el actual ordenamiento, en consonancia con la doctrina del derecho internacional de los derechos humanos, ha venido a proponer. Como hemos dicho, la no regulación no implica prohibición. Sin embargo, la falta de regulación genera desprotección.

Ante los numerosos proyectos legislativos recientemente presentados con el fin de regular la GS y los 23 fallos judiciales resueltos favorablemente, continuar acallando la técnica no impide que la misma se siga llevando a cabo. La sanción de la ley especial resulta urgente e impostergable, en el marco de una sociedad que ha decidido sancionar un código de fondo basado en el principio rector de igualdad y no discriminación.

Por otro lado, también resulta necesaria la contemplación de una legislación especial que se aboque a los casos de FPM. Si bien las situaciones planteadas son minoritarias, la FPM constituye un tipo de TRHA, las cuales están reguladas en el nuevo ordenamiento jurídico como la tercera fuente filial.

A diferencia de la GS, en la actualidad no constan proyectos legislativos presentados sobre FPM en nuestro país. Consideramos que una eventual regulación sobre el tema debe incluir el tratamiento jurídico no solo de embriones sino también de los gametos, en consonancia con la jurisprudencia de la Corte Interamericana de Derechos Humanos.

El trabajo en análisis viene a revelar cómo a dos años de entrada en vigencia del CCyCN, a pesar de la falta de regulación en materia de GS y FPM, la justicia ha saldado el vacío legislativo a través de sus resoluciones, volviendo al Código desfasado en relación con la realidad. Por todo ello, reafirmamos la necesidad de regulación especial, en tanto el silencio normativo en materia de derechos sustanciales no hace otra cosa que desproteger.

6 -Jurisprudencia (en el orden en que es citada) 
Ditieri, M.; Cortese, G.B. y González Demaría, Y. G. Cuando la realidad supera la norma: gestación por sustitución y filiación post mortem. Los efectos de su omisión. Derecho y Ciencias Sociales. Abril 2018. N 18. (Las familias y el derecho de las familias a dos años de vigencia del Código Civil y Comercial) Pgs 62-81 ISNN 1852-2971. Instituto de Cultura Jurídica y Maestría en Sociología Jurídica. FCJ y S. UNLP

Juzgado de Familia Nro. 7 de Lomas de Zamora “ B.J.D y otros s/ materia a categorizar” de fecha 30/11/2016. Disponible en http://www.villaverde.com.ar/es/sentencias/b-j-d-y-otross-materia-a-categorizar-277-exp-n-lz-52635-2016-juzgado-de-familia-nro-7-de-lomas-dezamora-30-11-2016/, recuperado el 20 de abril de 2018.

Cámara de Apelaciones en lo Civil, Comercial y Laboral Gualeguaychú, “B., M. A. c. F. C., C. R.”, 14/04/2010, cita La Ley online: AR/JUR/75333/2010. Juzgado de Familia de Gualeguay, "B. M. A. c/ F. C. C. R. | ordinario", 19/11/2013. Cita Microjuris online MJJU-M-83567-AR | MJJ83567.

Juzgado nacional en lo Civil $\mathrm{N}^{\circ}$ 86, "N.N. o DGMB s/ inscripción de nacimiento", 18/06/2013, cita Microjuris: MJ-DOC-6401-AR | MJD6401.

Tribunal de Familia N 7 de Rosario, "F.M. L y otra s/autorización judicial", 2/12/2014, Cita online AR/JUR/90178/2014.

Juzgado Nacional en lo Civil N $\mathrm{N}^{\circ}$ 102, “C., F. A. y otro c/ R. S., M. L. s/impugnación de maternidad”, 18/05/2015, en ABELEDO PERROT No: AR/JUR/12711/2015.

Juzgado Nacional Civil $\mathrm{N}^{\circ}$ 83, "NN O, s/inscripción de nacimiento", 30/06/2015, en http://colectivoderechofamilia.com/categoria/jurisprudencia/jurisprudencia-nacional, recuperado el 20 de abril de 2018.

Juzgado de Familia N 1 de Mendoza, "A. V. O., A. C. G. Y J. J. F", 29/7/2015, Cita online http://www.colectivoderechofamilia.com/wp-content/uploads/2015/07/FA.-PCIAL.PRIMER-JUZ.-FLIA.-MENDOZA.-Gestaci\%C3\%B3n-por-sustituci\%C3\%B3n..pdf, recuperado el 20 de abril de 2018.

Juzgado de Familia $\mathrm{N}^{\circ} 1$ de Mendoza, "C.M. E. Y J. R. M. POR INSCRIP. NACIMIENTO.”, 15/12/2015, en http://colectivoderechofamilia.com/fa-pcial-juz-flia-no1mendoza-gestacion-por-sustitucion, recuperado el 20 de abril de 2018.

Juzgado de Familia № 9 de San Carlos De Bariloche. "Dato Reservado. Expte. Nro. 10178 14.” Infojus: NV13851. 29 -12- 2015. Disponible en http://www.infojus.gob.ar/maternidadsubrogada-autorizan-transferencia-embrion-vientre-subrogado-nv13851-2015-1229/123456789-0abc-158-31ti-lpssedadevon, recuperado el 20 de abril de 2018.

Juzgado Familia nro. 7, Lomas de Zamora, "H. M. Y OTRO/A S/MEDIDAS PRECAUTORIAS (art.232 del CPCC)", 30/12/2015, en Microjuris online, MJ-JU-M97208-AR.

Tribunal Colegiado de Familia N 5 de Rosario, "S G G. y OTROS S/ FILIACION", 27/05/2016. Disponible en http://maestrosdelderecho.com.ar/jurisprudencia-gestacion-porsustitucion, recuperado el 20 de abril de 2018

Juzgado Nacional Civil Nro. 7, “A. R., C y otros c/ C., M. J. s/impugnación de filiación”, 23/05/2016, Inédito.

Juzgado Unipersonal de Familia No 2 de Moreno, "S. P., B. B. c/S. P., R. F. s/materia a categorizar", 4/07/2016, inédito. 
Ditieri, M.; Cortese, G.B. y González Demaría, Y. G. Cuando la realidad supera la norma: gestación por sustitución y filiación post mortem. Los efectos de su omisión. Derecho y Ciencias Sociales. Abril 2018. № 18. (Las familias y el derecho de las familias a dos años de vigencia del Código Civil y Comercial) Pgs 62-81 ISNN 1852-2971. Instituto de Cultura Jurídica y Maestría en Sociología Jurídica. FCJ y S. UNLP

Juzgado Nacional en lo Civil $\mathrm{N}^{\circ}$ 8, "B., B. M. y otro c/ G., Y. A. s/impugnación de filiación", 20/09/2016. Disponible http://www.colectivoderechofamilia.com/fa-nac-juz-nacciv-no-8-trha-gestacion-por-sustitucion, recuperado el 20 de abril de 2018.

Juzgado de Familia N³ de Gral. San Martín, “M., I. M. y otro s/autorización judicial, 22/08/2016, inédito.

Juzgado de Familia No 12 de Lomas de Zamora, "G. M., C y otro c/ W. B., A. V s/rectificación de partida", 3/10/2016, inédito.

Juzgado Familia $N^{\circ}$ 7, Lomas de Zamora, "B. J. D. y otros s/materia a categorizar", 30/11/2016, Inédito.

Juzgado Nacional en lo Civil No 81, "S., I. N. y otro c/A., C. L. s/Impugnación de Filiación", 14/06/2017, Inédito.

Juzgado de Familia N ${ }^{\circ} 7$ de Viedma, "reservado s/ autorizacion judicial (f)", 7/07/2017, Revista Jurídica Argentina La Ley. Buenos Aires. La Ley de fecha 22/08/2017, p. 7.

Juzgado de Familia $\mathrm{N}^{\circ} 1$ de Mendoza, "M.M.C y M.G.J. y R.F.N. por medidas autosatisfactivas", 6/09/2017, inédito.

Juzgado Nacional en lo Civil N 4, “S. T., V. s/inscripción de nacimiento", 20/10/2017, inédito.

Juzgado de Familia Na 3, Córdoba, "R., L. S. y otros - solicita homologacion" (EXPTE. N ${ }^{\circ}$ 3447358), 22/11/2017. Disponible en http://www.colectivoderechofamilia.com/fa-pcialjuz-flia-cordoba-trha-gestacion-por-sustitucion, recuperado el 20 de abril de 2018.

Tribunal Colegiado de Familia N $\mathrm{N}^{\mathrm{a}} 7$ Rosario, 2, "H., M.E. Y OTROS S/ venias y dispensas", 5/12/2017. Disponible en http://www.colectivoderechofamilia.com/wpcontent/uploads/2015/11/FA.-PCIAL.-TRIB.-COLEG.-FLIA.-N\%C2\%BA7.-ROSARIO-

SANTA-FE.-Gestaci\%C3\%B3n-por-sustituci\%C3\%B3n..pdf, recuperado el 20 de abril de 2018.

Tribunal de Familia de Morón no 3, “G.A.P. s/autorización”, 21/11/11. RDF 2012-III-119, AP/JUR/289/2011; Juzgado Nacional de Primera Instancia en lo Civil n ${ }^{\mathbf{0}} 3$, "K., J. V. c/Instituto de Ginecología y Fertilidad y otros s/amparo", 03/11/14. La Ley. AR/JUR/53958/2014.

Juzgado de Primera Instancia en lo Civil, Comercial, Laboral y de Minería no 4 de Santa Rosa, La Pampa, "A., C. V. c/Instituto de Seguridad Social-SEMPRE s/amparo", 30/12/2015. DFyP 2016 (agosto). AR/JUR/87457/2015.

Tercera Cámara de Apelaciones en lo Civil, Comercial, Minas, de Paz y Tributario de Mendoza, en Mendoza, "S.M.C. s/medida autosatisfactiva", 07/08/2014. RC J 6303/14. Juzgado Nacional de Primera Instancia en lo Civil n 87, "N.O.C.P. s/autorización", 05/05/2016. La Ley Online. Cita digital. AR/JUR/32492/2016. 
Ditieri, M.; Cortese, G.B. y González Demaría, Y. G. Cuando la realidad supera la norma: gestación por sustitución y filiación post mortem. Los efectos de su omisión. Derecho y Ciencias Sociales. Abril 2018. № 18. (Las familias y el derecho de las familias a dos años de vigencia del Código Civil y Comercial) Pgs 62-81 ISNN 1852-2971. Instituto de Cultura Jurídica y Maestría en Sociología Jurídica. FCJ y S. UNLP

\section{Bibliografía}

AAVV. (2015). Conclusiones de la Comisión Nro. 6. Derecho de Familia de las XXV Jornadas Nacionales de Derecho Civil. Disponible en http://jndcbahiablanca2015.com/wpcontent/uploads/2015/10/CONCLUSIONES-06.pdf, recuperado el 20 de abril de 2018.

Comisión de Reformas (2012) Proyecto de Código Civil y Comercial de la Nación, disponible en http://www.nuevocodigocivil.com/wp-content/uploads/2015/02/5Fundamentos-del-Proyecto.pdf, recuperado el 20 de abril de 2018.

Honorable Congreso de la Nación (2012) Dictamen de la Comisión Bicameral para la reforma, actualización y unificación de los códigos civil y comercial de la Nación. Disponible en : http://www.cabb.org.ar/noticias/proye.pdf, recuperado el 20 de abril de 2018.

Herrera, M. (2016). El consentimiento informado prestado desde el más allá y presumido en el más acá. A raíz de un resonado fallo sobre filiación post mortem. Diario DPI Suplemento Derecho Civil, Bioética y Derechos Humanos, 2016 (14), p 3-4.

Herrera M. y Lamm E. (2014). Arts. 509 a 593. Kemelmajer de Carlucci A., Herrera M. y Lloveras N. Tratado de Derecho de Familia Según el Código Civil y Comercial. Tomo II. Santa Fé. Rubinzal - Culzoni Editores. P. 531

Merlo L. (2013). El proyecto de unificación de los Códigos Civil y Comercial en materia de derecho de familia tras la media sanción del Senado. Microjuris, cita online: MJ-DOC6536-AR

Pérez A. (2014). Fertilización Post Mortem: Qué dicen y que piensan los medios y la doctrina en nuestro país. AbeledoPerrot. AP/DOC/2832/2013. p 6.

Rodríguez Iturburu M. (2018). Gestación por sustitución: las cosas por su nombre, ni alquiler de vientre ni maternidad subrogada. Estado actual en la Argentina. Diario DPI suplemento Civil, Bioética y Derechos Humanos nro. 34, 21-02-2018, disponible en https://dpicuantico.com/sitio/wp-content/uploads/2018/02/Iturburu-Civil-Bioetica-yDerechos-Humanos-21.2.pdf. 